\title{
Using Anomalous Dispersion Effect for Fourier Transform Analysis of X-ray Reflectivity from Thin-Film Stacks
}

\author{
Kazuhiro Ueda \\ Advanced Research Laboratory, Hitachi, Ltd., HATOYAMA, SAITAMA, 350-0395, JAPAN \\ Fax: 81-49-296-5999, e-mail: kazuhiro.ueda.ha@hitachi.com
}

\begin{abstract}
We used the anomalous dispersion effect and a Fourier transformation method for analysis of $\mathrm{X}$-ray reflectivity without using theoretical layer models. The reflectivity of a layer stack was measured at two X-ray wavelengths, the near $\mathrm{Ru}-\mathrm{K}$ edge and pre Ru-K edge, for a sample with a $0.8 \mathrm{~nm}$ thick $\mathrm{Ru}$ layer at the stack top. We calculated the differential reflectivity between the two wavelengths by subtracting the near Ru-K edge curve from that of the pre $\mathrm{Ru}-\mathrm{K}$ edge. The result of fast Fourier transformation (FFT) performed on the differential curve showed interface depths from the sample surface to the interface. This result suggests the promising candidate for analyzing the layer structure of a sample by using the anomalous dispersion effect and FFT methods.
\end{abstract}

Key words: Anomalous dispersion, Fourier transform, x-ray reflectivity

\section{INTRODUCTION}

Increasingly, thin-layered stack materials with layer thickness in the nanometer range are being used in modern technology and industry (e.g., microelectronics and magnetic devices). Especially, giant magnetoresistive (GMR) spin-valve heads have been investigated for application to high-recording-density hard drives because these heads have high sensitivity for reading magnetic records [1]. Recently, many researchers have studied using perpendicular magnetic-recording media [2] and advanced spin-valve heads with magnetic tunnel junctions for advanced hard drives [3]. The GMR structures consist of two ferromagnetic layers separated by a noble metal spacer with a thickness of a few nanometers. The GMR structures were deposited on antiferromagnetic PtMn thin layer. Their magnetic properties strongly depend on the thickness of each layer.

$\mathrm{X}$-ray reflectivity is a powerful tool for investigating layer thickness, electron density, and interface roughness [4-6]. For layered materials, $x$-ray at grazing incidence are reflected and transmitted at each interface. Because of the interference between the x-rays reflected at the various interfaces, fringes are seen in the reflectivity. These oscillations of reflectivity curve reveal the thicknesses of different layers. The least-squares method with theoretical models has usually been used for analysis of reflectivity [7-8]. However, to obtain a best-fit result for a measured reflectivity curve is difficult unless the thicknesses of all the layers in a sample are known. Fourier transform analysis of the reflectivity can determine each layer thickness [9] Nevertheless, the Fourier transform does not allow determination of the exact order of stacking. The Wavelet transform analysis does show that analyzing the order of stacking is possible [10]. Even so, using the Fourier transform and the Wavelet transform to analyze layered stacks with similar thicknesses is difficult.

Therefore, we investigated layer thicknesses and the order of stacking with the Fourier transform analysis. We then used the anomalous-dispersion effect in this Fourier transform analysis to obtain reflectivity curves.

\section{EXPERIMENTS}

Two samples were sputtered onto a five-inch diameter silicon disk in a static magnetic field. The first sample (A) was deposited on the silicon disk in the order tantalum (Ta) (10 nm thick), platinum-manganese (PtMn) $(10 \mathrm{~nm})$, and ruthenium $(\mathrm{Ru})(0.8 \mathrm{~nm})$, the cap layer of the sample. The other sample (B) was deposited on the silicon disk in the order Ta $(10 \mathrm{~nm})$, PtMn (15 $\mathrm{nm})$, Ta $(10 \mathrm{~nm})$, and $\mathrm{Ru}(0.8 \mathrm{~nm})$. The numbers in parentheses are nominal thicknesses. The reflectivity curves were measured at two energies with a diffractometer at BL16XU in SPring-8 [11].

The incidence $\mathrm{X}$-ray energies were the near Ru K-edge $(22.09 \mathrm{keV})$ and the pre Ru K-edge $(21.88 \mathrm{keV})$. They were measured using $\theta-2 \theta$ scanning technique and simultaneously transformed from incident angles to scattering vectors. The reflectivity analysis consists of two steps. In the first step, we calculate the differential reflectivity curve by subtracting the near $\mathrm{K}$-edge curve from the pre K-edge one. In the second step, we transform the differential reflectivity curve by using the first Fourier transform (FFT) method. The result of the FFT shows the interface depth of the measured sample.

\section{RESULTS AND DISCUSSION}

\subsection{Analysis of Differential Reflectivity}

Figure 1 shows the reflectivity curves of sample (A) at two energies and the differential reflectivity curve obtained by subtracting the near-edge curve from that of the pre-edge. The two curves of reflectivity look the same. The differential result reveals fringes in the differential reflectivity curve. The fringes of reflectivity curve show the interference between surface and interface of $\mathrm{PtMn} / \mathrm{Ta}$, surface and interface of $\mathrm{Ta} / \mathrm{Si}$, and interfaces of $\mathrm{PtMn} / \mathrm{Ta}$ and $\mathrm{Ta} / \mathrm{Si}$. Using the abnormal 


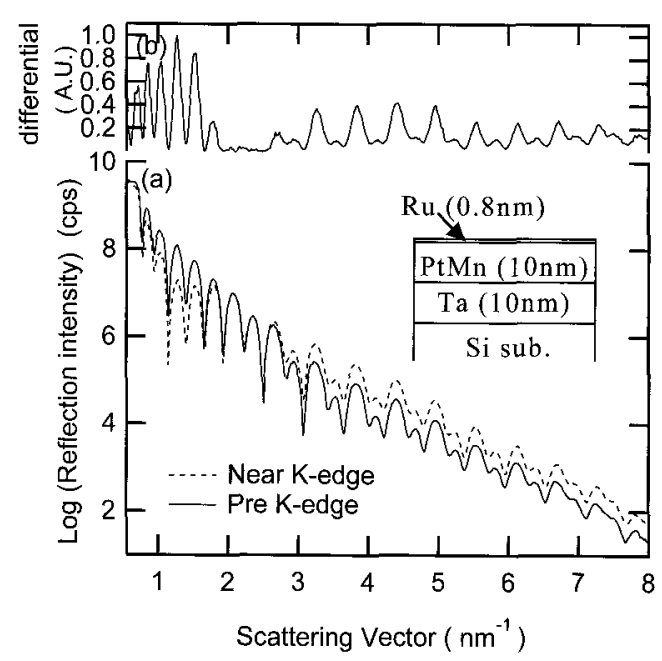

Fig. 1 Experimental reflectivity measured at two energies for sample (A) with a $10 \mathrm{~nm}$ thick PtMn and Ta layers.

(a): Reflectivity curves measured at two energies. (b): Differential reflectivity obtained by subtraction of two energy curves.

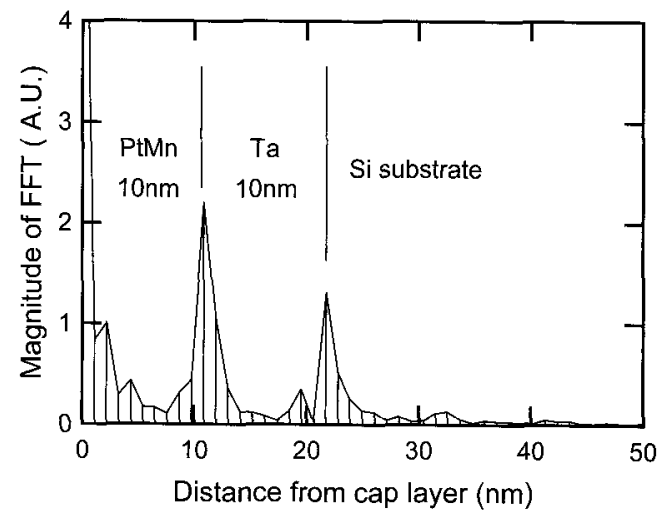

Fig. 2 The Magnitude of the FFT obtained from the differential reflectivity curve. Peaks are found at 10.5 , and $21.4 \mathrm{~nm}$.

dispersion of the surface layer can change only the oscillation of the reflection from the surface layer. The interference fringe that is between interfaces of $\mathrm{PtMn} / \mathrm{Ta}$ and $\mathrm{Ta} / \mathrm{Si}$ is subtracted by the near K-edge reflectivity from the pre K-edge one. Figure 2 shows the magnitude of the fast Fourier transform (FFT) of differential reflectivity. Peaks are found at 10.5 , and $21.4 \mathrm{~nm}$. The peak positions agree well with the distance from the $\mathrm{Ru}$ layer.

The reflectivity curve of the pre K-edge was analyzed using best fitting with the calculated reflectivity curve. The fitting result revealed the depth of the interfaces $(\mathrm{PtMn} / \mathrm{Ta}$ and Ta/Si substrate) to be 10.22 , and $20.97 \mathrm{~nm}$ This suggests the differential reflectivity curve reveals the thicknesses of different layers.

\subsection{Interface depth information}

Figure 3 shows the reflectivity curves of sample (B) at two energies and the differential reflectivity curve obtained by the subtraction of the near-edge curve from that of the pre-edge. The differential reflectivity shows only the interference of reflection waves from the $\mathrm{Ru}$ interface and the other interfaces. These oscillations of the reflectivity curve reveal the distance from the $\mathrm{Ru}$ layer.

Figure 4 shows the magnitude of the FFT of differential reflectivity. Peaks are found at 11.4, 25.0, and $34.6 \mathrm{~nm}$, but not at $15 \mathrm{~nm}$. The peak positions agree well with he distance from the Ru layer, not the layers thicknesses. Table I shows the result of differential reflectivity obtained using the FFT.

The reflectivity curve of the pre K-edge was analyzed by best fitting with the calculated reflectivity curve. The results of best fitting are also listed in Table I. The fitting result revealed that the Ta layer was oxidized from a depth of $2.3 \mathrm{~nm}$. However, the oxide layer was not analyzed with differential reflectivity. The information from the Table I suggests that the minimum depth of the interface from the surface that can be analyzed is about $5 \mathrm{~nm}$ under these experimental conditions.

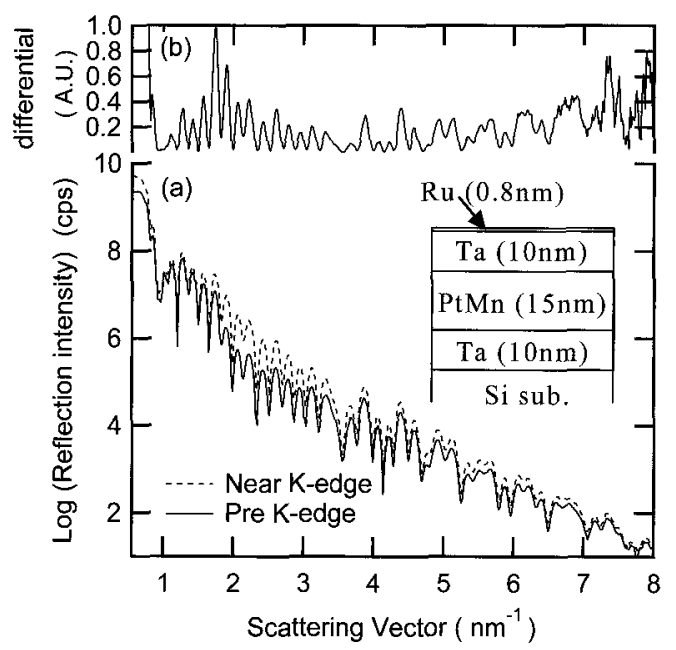

Fig. 3 Experimental reflectivity measured at two energies for sample (B) with a $15 \mathrm{~nm}$ thick PtMn layer. (a): Reflectivity curves measured at two energies. (b): Differential reflectivity obtained by subtraction of two energy curves.

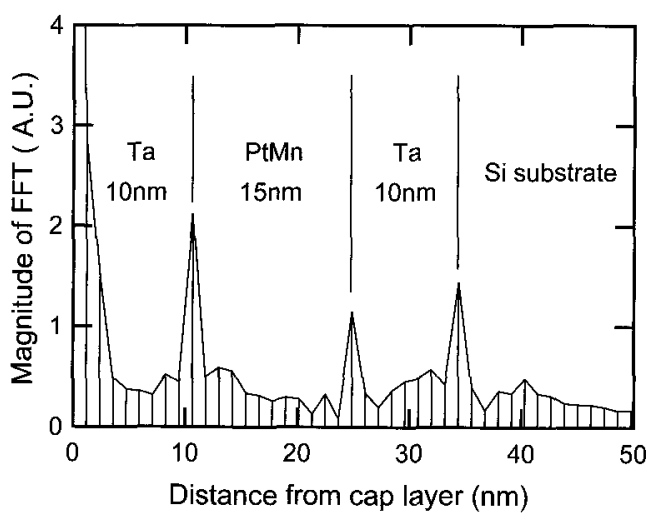

Fig. 4 The Magnitude of the FFT obtained from the differential reflectivity curve. Peaks are found at 10,25, and $35 \mathrm{~nm}$ but not at $15 \mathrm{~nm}$. The peak positions agree well with the distance from the Ru layer. 
Table I Layer thickness in the sample (B) determined by best fit analysis and differential reflectivity method.

\begin{tabular}{lcc}
\hline Materials & $\begin{array}{c}\text { Differential } \\
\text { reflectivity analysis } \\
\text { (nm) }\end{array}$ & $\begin{array}{c}\text { Best-fit } \\
\text { analysis } \\
\text { (nm) }\end{array}$ \\
\hline $\mathrm{Ru}$ & - & 0.75 \\
$\mathrm{TaOx}$ & - & 2.33 \\
$\mathrm{Ta}$ & 11.4 & 9.24 \\
$\mathrm{PtMn}$ & 13.6 & 13.90 \\
$\mathrm{Ta}$ & 9.6 & 10.02 \\
$\mathrm{Si}$ & Substrate & Substrate \\
\hline
\end{tabular}

\section{CONCLUSION}

These results suggest the strong possibility that the layer structure in measured samples can be analyzed using the anomalous dispersion effect and FFT methods. The stack information is revealed by the distance from the cap layer to the interface. The minimum depth of the interface from the surface that can be analyzed under these experimental conditions is about $5 \mathrm{~nm}$. Further study will show how to extract the stack information from the differential reflectivity profile by using the anomalous dispersion effect of Fourier transform analysis.

Acknowledgments. The authors thank Drs. Y. Hirai (ARL, Hitachi, Ltd.) and T. Hirano (HRL, Hitachi, Ltd.) for their useful discussions on the research. The authors also thank Dr. K. Watanabe (CRL, Hitachi, Ltd) for conducting the experimental measurements.
References

[1] B. Dieny, V. S. Speriosu, S. S. Parkin, B. A. Gurney, D. R. Wilhoit and D. Mauri, Phys. Rev. Lett., B43, 1297 (1991).

[2] M. Futamoto, Y. Hirayama, Y. Honda, K. Itoh and K. Yoshida, J. Magn. Soc. Jpn., 21, 141 (1997).

[3] J. S. Moodera and G. Mathon, J. Magn. Magn Mater., 200, 248 (1999).

[4] T. C. Huang, J. -P. Nozieres, V. S. Speriosu, J. Lefakis and B. H. Gurney, Appl. Phys. Lett., 60, 1573 (1992).

[5] K. Usami, K. Ueda, T. Hirano, H. Hoshiya and S. Narishige, J. Magn. Soc. Jpn., 21, 441 (1997)

[6] T. Hirano, K. Usami, K. Ueda and H. Hoshiya, J Synchrotron Rad., 5, 969 (1998).

[7] L. G. Paratt, Phys. Rev., 95, 259 (1954).

[8] K. S. Sinha, E. B. Sirota and S. Garoff, Phys. Rev., B38, 2297 (1988).

[9] K. Sakurai and A. Iida, Jpn. J. Appl. Phys. 31, L113 (1992).

[10] E. Smigiel and A. Cornet, J. Phys. D: Appl. Phys., 33, 1757 (2000).

[11] Ya. Hirai, S. Yasuami, A. Kobayashi, Yo. Hirai, J. Nishino, M. Shibata, K. Yamaguchi, K. Y. Liu, S.

Kawado, T. Yamamoto, S. Noguchi, M. Takahashi, I.

Konomi, S. Kimura, M. Hasegawa, N. Awaji, S. Komiya, T. Hirose, S. Ozaki, T. Okajima, T. Ishikawa and H. Kitamura, Nucl. Instr. and Meth., A521, 538 (2004).

(Received December 9, 2006;Accepted January 26, 2007) 